\title{
ELECTRODIAGNOSTIC TESTS IN EYE DISEASE
}

\author{
JOAN BEHRMAN, B.Sc. \\ Electrodiagnostic Clinic, Moorfields Eye Hospital, London.
}

Electrodiagnostic tests such as the ECG and EEG are now widely used in medicine. Similarly, in Ophthalmology, the Electroretinogram (ERG) and the Electro-oculogram (EOG) are becoming of increasing importance in the diagnosis of eye disease.

\section{Basis of the Electroretinogram}

An electrical potential exists between the cornea and the fundus of the living eye. When the eye is illuminated by a flash of light a small potential is generated in addition to this corneofundal potential, which forms the ERG. This is analogous to the way in which the ECG is recorded above the steady skin potential. The final response is the sum of a number of components arising in the different neural layers of the retina and consists primarily of an initial negative a-wave, followed by a positive b-wave. A contact lens electrode and an indifferent electrode placed on the skin near the eye record the ERG. Diseases which affect the receptor cells or the second order neurones, the bipolar cells, will alter the size and shape of the ERG, (Jacobson, 1961).

\section{Basis of the Electro-Oculogram}

The EOG is concerned with the slower changes in the standing potential of the eye rather than the rapid activity of the retinal neurones which produces the ERG. This standing potential can be measured using the "eye movement technique" in which electrodes are placed on either side of the eye and the eye moved from side to side. The potential difference induced between the two electrodes is then proportional to the standing potential on the eye and is recorded.

In the clinical test, there is an initial drop in the standing potential during a period of dark adaption, followed by an increase in potential occuring over several minutes when the eye is subsequently exposed to light. This is the basis of the EOG (Arden, Barrada and Kelsey, 1962). The changes in the potential which occur when the retinal illumination is altered appear to be related to the metabolism of the pigment epi- thelium. Any disease therefore which affects the rods or the pigment epithelium will give an abnormal EOG result.

\section{Uses of Electrodiagnostic Tests}

The ERG and EOG provide objective tests of retinal function. Since they are concerned with different layers of the retina, the ERG with the first and second order neurones and the EOG with the pigment epithelium and the first order neurones, they complement each other. Some conditions will affect the results of one test only, others the results of both. Disturbances of vision due to diseases of the optic nerve, however, will not cause any electrodiagnostic abnormality.

\section{Retinal Detachment}

In retinal detachment the EOG immediately becomes grossly abnormal and there is no corresponding rise in potential when the illumination is increased. The size of the b-wave in the ERG is thought to be reduced in proportion to the area of the detachment, and the ERG is extinguished altogether if the retina dies (Rendahl, 1957). Electrodiagnostic tests are of importance when a detachment is suspected and the fundus cannot be viewed directly due to the presence of cataract or corneal opacities.

\section{Tapeto-Retinal Degenerations}

Electrodiagnostic tests can also be of assistance in diagnosing and distinguishing between the various types of retinal degeneration. In retinitis pigmentosa, for instance, the ERG is very small and in severe cases non-recordable. The EOG is more sensitive. however, and is pathological even before ERG or fundus changes becomes manifest (Arden and others, 1962). These tests thus provide a method of early diagnosis. especially useful in hereditary diseases of this kind.

\section{Vascular Lesions}

In an occlusion of the central retinal vein or one of its branches, the EOG in most cases shows a reduced light rise, and both the size 
and waveform of the ERG are affected. Electrodiagnostic tests are found to be of prognostic value here; a normal EOG and an ERG in which the b-wave is not diminshed indicating a good prognosis as far as recovery of visual function is concerned (Karpe and Germanis, 1961).

\section{Myopia}

A low EOG result is obtained in certain cases of degenerative myopia, probably because the earliest degenerative changes occur in the pigment epithelium. Later on the ERG is also affected and the typical fundus picture is seen. Electrodiagnostic tests are of assistance here in diagnosing progressive myopia in early cases or in childhood.

\section{Choroidal Lesions}

In conditions such as acute choroiditis which affect the outer layers of the retina only, an abnormal EOG will be obtained, although in most cases the ERG remains unaffected.

\section{Vitamin A Deficiency}

Vitamin A deficiency may induce ocular changes including night blindness. The waveform of the ERG is affected and an abnormal EOG is also recorded. This usually returns to normal when the metabolic error is corrected, provided the deficiency is not prolonged, in which case permanent damage may occur.

\section{Drug Retinopathies}

Certain drugs, such as chloroquine, an antimalarial used today in the treatment of collagen diseases, have been found to induce retinal changes which may lead to a retinopathy if the drug is not withdrawn. Electrodiagnost造 tests detect these changes in the eart stages when they are still reversible, and the EOG in particular gives a reduces light rise before any visible signs appea? in the fundus (Arden, Friedman and Kolb: 1962). If the tests are repeated at regular inters vals, together with an ophthalmological exang ination, the drug therapy can be stopped wher the EOG falls below a certain level and a res tinopathy can be prevented.

\section{Summary}

Electrodiagnostic tests are of most value t $\vec{\theta}$ the clinician in the following situations:

1. In cases where ophthalmoscopic observå tion is precluded due to the presence of opacities in the media.

2. When subjective tests are unreliable duẹ to the age of the patient.

3. In prognosis in cases of vascular disorders?

4. In early diagnosis of hereditary or con: genital diseases.

5. In prevention of disease, as in toxic re $\mathrm{D}^{\frac{\mathbb{Z}}{3}}$ tinopathies.

6. In providing information in some of conditions where there are no physical sims of eye disease.

It must be realised, however, that electrodiag nostic tests will give abnormal results onl in generalised retinal disorders. A localise lesion affecting the macula, for instance, will noe be detected.

\section{PEFERENCES}

Arden, G. B., Barrada, A., and Kelsey, J. H. (1962): New Clinical Test of Retinal Function based upon the Standing Potential of the Eye, Brit. J. Ophthal., 46, 449.

Arden, G. B., Friedman, A., and Kolb, H. (1962): Anticipation of Chloroquine Retinopathy, Lancet, i, 1164. JACOBSON, J. H. (1961): "Clinical Electroretinography." Springfield, Illinois: Charles C. Thomas.

Karpe, G., and Germanis, M. (1961): The Prognostic Value of the Electroretinogram in Thrombosis of the Retinal Veins, Acta ophthal. (Kbh.), Supp. 70.

Rendahl, I. (1957): The ERG in Detachment of the Retina, A.M.A. Arch. Ophthal., 57, 566. 